\title{
Análisis comparativo de las propiedades mecánicas de un material compuesto reforzado con fibras de carbono y las de su matriz polimérica de resina epóxica
}

\author{
Comparative analysis of the mechanical properties \\ of a composite material reinforced with carbon \\ fibers and the polymer matrix of epoxy resin
}

\author{
Anabel Nava Gastelum ${ }^{1}$, Miriam Siqueiros Hernández ${ }^{2}$, \\ Benjamín Gonzalez ${ }^{2}$, Yuridia Vega ${ }^{2}$, Ismael Mendoza Muñoz ${ }^{3}$
}

\footnotetext{
${ }^{1}$ Escuela de la Ciencia de la Ingeniería y Tecnología (ECITEC), Universidad Autónoma de Baja California, Blvd. Universitario \#1000, Unidad Valle de las Palmas, Tijuana Baja California, México e-mail: anabel.nava@uabc.edu.mx

${ }^{2}$ Escuela de la Ciencia de la Ingeniería y Tecnología (ECITEC), Universidad Autónoma de Baja California, Blvd. Universitario \#1000, Unidad Valle de las Palmas, Tijuana Baja California, México e-mail: miriam.siqueiros@uabc.edu.mx; bgonzalez79@uabc.edu.mx; vegay@uabc.edu.mx

${ }^{3}$ Universidad Autónoma de Baja California, Facultad de Ingeniería, Blvd. Benito Juárez s/n Col: Ex Ejido Coahuila, Mexicali Baja California, México.

e-mail: ismael.mendoza@uabc.edu.mx
}

\section{RESUMEN}

En los últimos años los materiales compuestos se han desarrollado para diferentes aplicaciones del área ingenieril. En este trabajo se expone primeramente un análisis de una matriz polimérica, en este caso resina epóxica para su aplicación en un material compuesto reforzado con fibras. Se presenta un estudio de las propiedades mecánicas, el cual se llevó a cabo mediante pruebas de tensión realizadas bajo el régimen de la norma ASTM D638; se manufacturaron cinco especímenes de resina los cuales llevaron un proceso de preparación de moldeo adecuado para la optimización de probetas tipo I. El objetivo fue conocer las propiedades de la matriz termoestable para así reforzarla con fibras de carbono y mejorar las propiedades del material compuesto. Posteriormente se analizaron las propiedades mecánicas del material compuesto en el cual se utilizó el refuerzo de fibra de carbono 3k. Para el análisis se fabricaron especímenes con una orientación [0/+45/45]s; las pruebas realizadas en los especímenes de material compuesto fueron cinco ensayos de tracción basados en la norma ASTM D3039. El proceso de manufactura empleado para la realización de los especímenes de material compuesto fue el método VACCUM BAG WET LAY-UP. Se comprobó que las probetas de matriz polimérica de resina epóxica generan burbujas por el mezclado y por el curado al ambiente; así mismo estas burbujas trabajan como concentradores de tensiones que debilitan el material en pruebas de tracción. En comparación con las propiedades mecánicas del material compuesto, estos muestran un mejor comportamiento por el refuerzo de fibra de carbono y por el proceso de manufactura al vacío.

Palabras clave: matriz polimérica, espécimen, refuerzo, termoestable, vaccum bag.

\section{ABSTRACT}

In recent years composite materials have been developed for different applications in engineering areas. This paper first presents the tensile properties of a polymer, in this case an epoxy resin, to be used as the matrix of a fiber reinforced composite material. A study of the mechanical properties is presented, which was carried out by performing tensile tests under the regime of ASTM D638; five resin specimens were manufactured in which a suitable molding process was performed to optimize specimens type I. The objective was to determine the properties of the thermoset matrix to be reinforced with carbon fiber to improve the composite properties. Subsequently, mechanical properties of the composite reinforced with carbon $3 \mathrm{k}$ fibers, are analyzed. For proper analysis ten specimens with an orientation $[0 /+45 /-45]$ s were made; five tensile tests were 
conducted in composite specimens based on ASTM standard D3039. The specimens manufacturing process followed the VACCUM BAG WET LAY-U method, under normal air. It is found that epoxy resin specimens generate bubbles by mixing and curing in air. These bubbles, working as stress concentrators, weaken the material tensile strength as compared to the mechanical response of the composite material, which certainly shows better performance due to reinforcing carbon fibers and the manufacturing process in vacuum.

Keywords: thermoset, matrix, specimens, vaccum bag, epoxy.

\section{INTRODUCCIÓN}

Actualmente el desarrollo de los materiales compuestos se basa en la necesidad de obtener materiales con mejores propiedades, en donde se combine una buena resistencia y tenacidad. De acuerdo con GUERRERO [1] la utilización de materiales compuestos está en un rápido crecimiento, implantándose en una gran variedad de sectores industriales, esto es gracias a sus magníficas propiedades mecánicas, así como su baja densidad; ya que con ellos se pueden obtener materiales ligeros, resistentes, dúctiles y resistentes a altas temperaturas. Como lo menciona GARCÍA CASTILLO [2] los materiales compuestos de fibra de carbono en matriz polimérica, tienen actualmente un amplio campo de aplicaciones, en la industria aeronáutica, automotriz y médica. Teniendo en cuenta que día a día crece la oportunidad de optimizar el diseño de materiales compuestos, se puede afirmar que es necesario conocer las propiedades mecánicas de los materiales que se van a construir, ya sea para su empleo en diferentes sectores industriales. En los últimos años las fibras de carbono han sido utilizadas en distintas aplicaciones, donde sus propiedades mecánicas y ligereza son muy importantes. Así mismo los materiales compuestos de matriz polimérica son cada día más empleados ya que presentan excelentes propiedades mecánicas respecto a una elevada relación resistencia-peso tal como lo comentan MENDOZA-NAVA et al. [3].

El presente estudio tiene como objetivo conocer a fondo las propiedades del material compuesto, así como las de su refuerzo y matriz termoestable, aplicando normas para su caracterización y para el proceso de manufactura de los especímenes de resina epóxica. Con el fin de comparar las propiedades del composite respecto con su matriz polimérica, y así comprobar que el incremento de las propiedades mecánicas del material compuesto reside más en el refuerzo empleado, en este caso fibra de carbono $3 \mathrm{~K}$. Ya que como afirma RAMOS [4] las fibras de carbono como material reforzante, reflejan mayor efectividad en el desvió de fracturas internas en los materiales compuestos, logrando que estas no sigan una trayectoria definida y a su vez logran agregan excelentes propiedades mecánicas al tener la capacidad de absorber energía.

\section{MATERIALES Y MÉTODOS}

\subsection{Materiales}

Los materiales empleados para los análisis de las propiedades mecánicas fueron: resina epóxica Fibre Glast $\mathrm{t}^{\mathrm{MR}}$ de baja viscosidad como matriz termoestable del material compuesto, así como para el análisis de probetas tipo I, cuenta con una densidad de $1.10-1.20 \mathrm{~g} / \mathrm{cm} 3$ para actuar con un catalizador a temperatura ambiente, presentando en la ficha técnica de sus propiedades un esfuerzo máximo de 340MPa para cargas de tensión. Como material de refuerzo del material compuesto se empleó fibra de carbono 3K (3000 filamentos por mechón), el tejido de la fibra ofrece una de las relaciones más altas de resistencia-rigidez, con una resistencia a la tracción de 4200-4370 MPa, atribuyendo su peso ligero y su baja densidad al material compuesto.

\subsection{Métodos}

\subsubsection{Elaboración de probetas tipo I}

Para la elaboración de especímenes se emplearon 210 gr de resina epóxica System 1000 y 21 gr de catalizador System 1025 para un tiempo de reacción de 25 minutos[5], utilizando un arreglo de resina y catalizador de 10:1. El proceso de mezcla empleado fue manual sin agitación mecánica .Esta resina se utiliza principalmente para la aplicación de materiales poliméricos reforzados de fibra, El curado de la resina fue a temperatura ambiente $\left(22^{\circ} \mathrm{C}\right.$ a $\left.25^{\circ} \mathrm{C}\right)$ de 24 a 48 horas sin utilizar un proceso de desgasificación al vacío.. Las probetas se manufacturaron bajo las especificaciones de la ASTM D638 [6], cumpliendo así con las medidas establecidas de la norma, gracias al uso de moldes de silicón para la obtención de la geometría adecuada, tal como se muestra en la figura 1. 


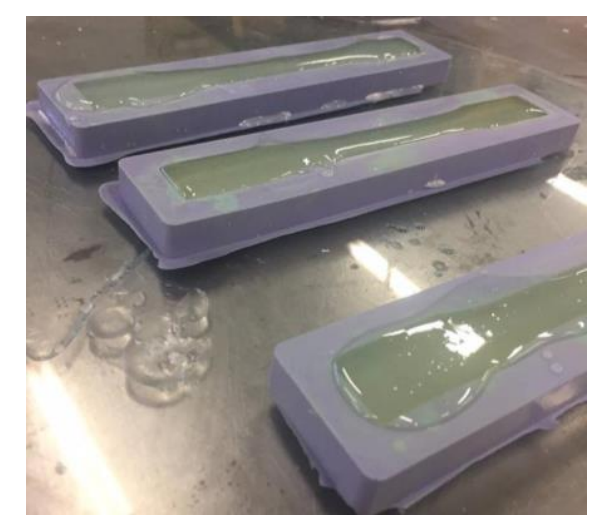

Figura 1: Resina epóxica antes del curado en moldes de silicón

Posteriormente, una vez que las probetas cumplieron son su tiempo de curado, se procedió a rectificarlas de manera manual como se muestra en la figura 2, se utilizaron lijas de diferentes tamaños de grano, comenzando del más grueso al más fino y cambiando de orientación de $0^{\circ}$ y $90^{\circ}$. Este proceso se llevó a cabo por el hecho que la resina epóxica al curarse a temperatura ambiente desarrolla una contracción de sus moléculas internas dejando así un acabado cóncavo superficial, el cual puede afectar en los ensayos de tensión por la sujeción que se lleva a cabo en el área de las mordazas.

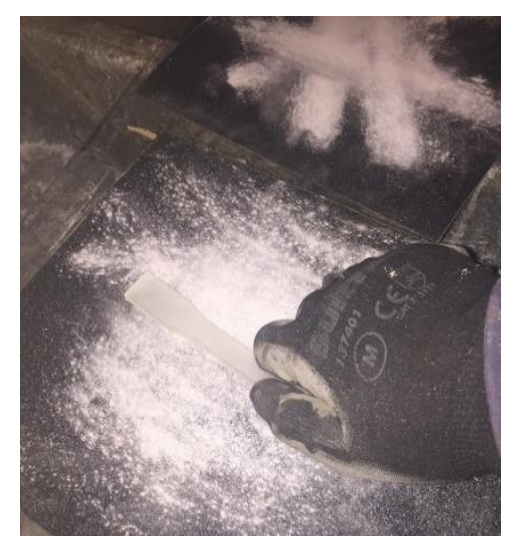

Figura 2: Rectificado de especímenes de resina epóxica con lijas a diferentes grados de orientación.

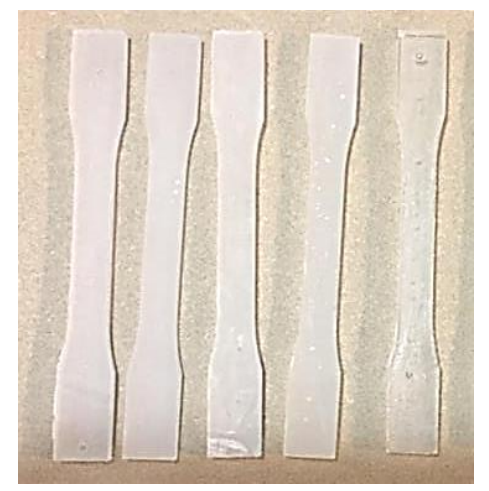

Figura 3: Probetas tipo I de resina epóxica. 


\subsubsection{Caracterización de especímenes de material compuesto}

Para la caracterización de material compuesto, se llevó a cabo el corte de 6 capas de fibra de carbono $3 \mathrm{~K}$ de espesor nominal de $0.30 \mathrm{~mm}$, con una orientación [0/₫45] s, el cual es un laminado simétrico balanceado cuasi-isotrópico. La fibra se impregno se su matriz termoestable con 150 gr resina epóxica y 15 gr de catalizador, utilizando el arreglo de mezclas 10:1, se aplicó el método de manufactura por vacío, como se muestra en la figura 4; este método es aplicado con una bomba vacío, la cual genera succión para impregnar las fibras con resina dentro del molde.

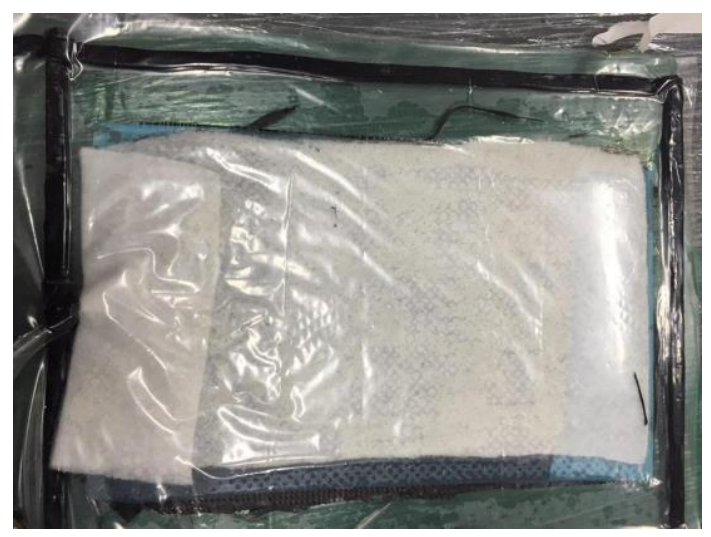

Figura 4: Proceso de manufactura al vacío para caracterización de material compuesto.

El tiempo de curado del material compuesto fue alrededor de 72 horas a temperatura ambiente, el método por vacío muestra un buen acabado superficial del composite, así como se muestra en la figura 5; posteriormente bajo las especificaciones de la norma ASTM D3039 [7] se cortaron 5 especímenes de material compuesto, así como se observa en la figura 6, el cual es el número que se requiere para la aplicación de pruebas de tensión, a continuación en la tabla 1 se muestran las medidas especificadas por ASTM D3039 para probetas de material compuesto.

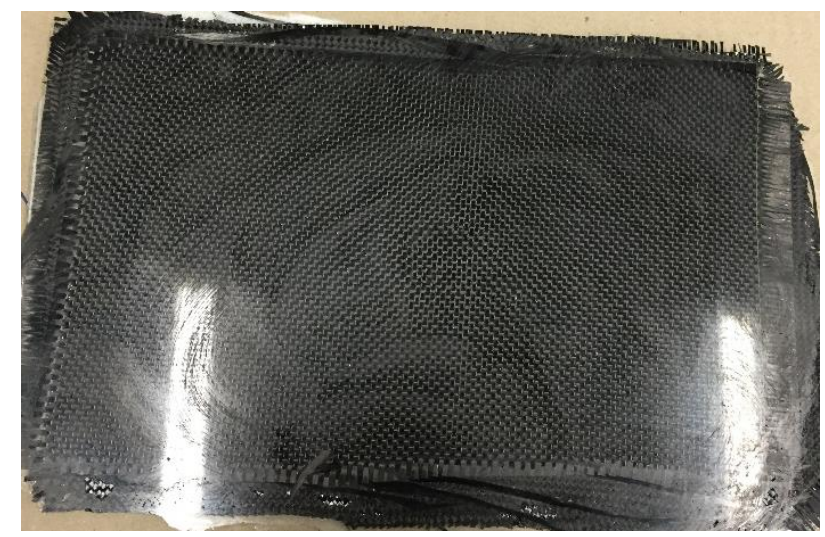

Figura 5: Laminado de Material compuesto.

Tabla 1: Geometría de especímenes recomendada por ASTM D3039 para pruebas de tensión.

\begin{tabular}{c|c|c|c}
\hline ORIENTACIÓN & ANCHO & LONGITUD & ESPESOR \\
\cline { 2 - 4 } DE LA FIBRA & $\mathrm{mm}[\mathrm{in}]$ & $\mathrm{mm}[\mathrm{in}]$ & $\mathrm{mm}[\mathrm{in}]$ \\
\hline $\begin{array}{c}\text { Simétrico y ba- } \\
\text { lanceado }\end{array}$ & $25[1,0]$ & $250[10,0]$ & $2,5[0,100]$ \\
\hline
\end{tabular}




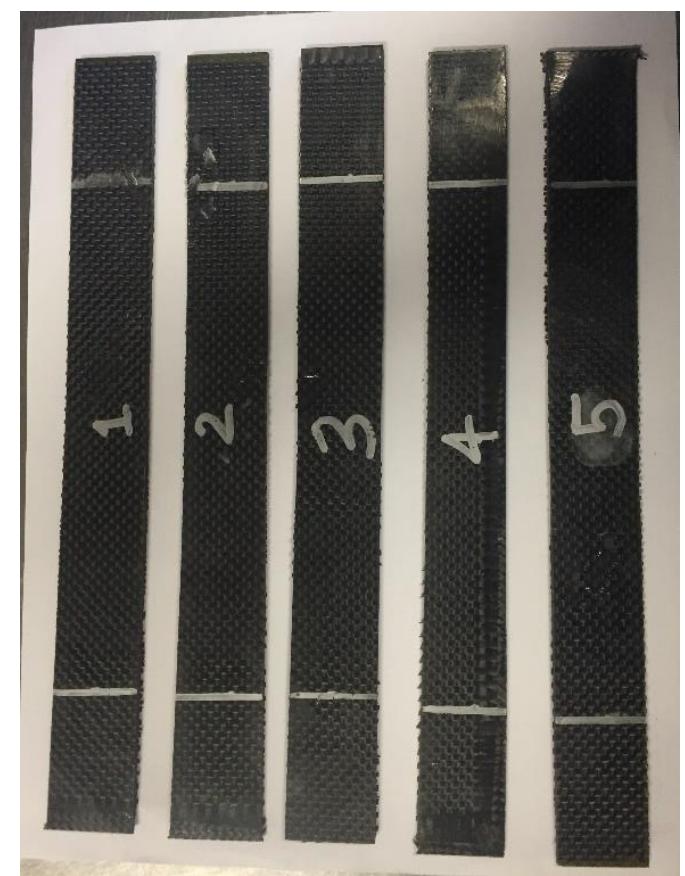

Figura 6: Especímenes de material compuesto, regidos bajo ASTM D3039.

\subsection{Procesos}

\subsubsection{Corte de material compuesto}

Para realizar el corte de las cinco probetas de material compuesto, se utilizó un cortador de un disco de metal tal como se muestra en la figura 7, con una velocidad de 3500 RPM y 550W de potencia, con esta herramienta el material compuesto no sufre alguno daño y se logra obtener una buena superficie de corte, tal como lo menciona SIQUEIROS et al. [8].

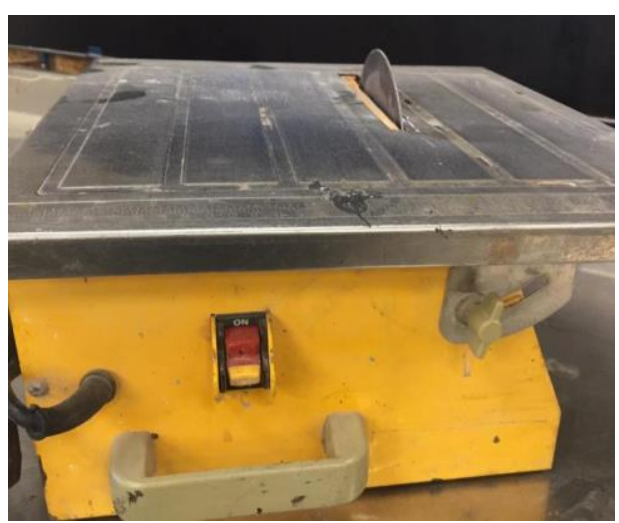

Figura 7: Cortador de disco de acero, utilizado para obtener probetas de material compuesto estandarizadas por mediadas de ASTM D3039

\subsubsection{Ensayos de tensión}

Para los ensayos de tensión se utilizó la maquina universal de ensayos SHIMADZU, cuenta con una celda de carga de $100 \mathrm{KN}$, la velocidad empleada para los especímenes fue de $2 \mathrm{~mm} / \mathrm{min}$ [9]., la cual es especificada en las normas ASTM D638 Y D3039.

Como se observa en la figura 8, los especímenes de resina epóxica sometidos a pruebas de tensión, muestran el resquebrajamiento localizado en la zona calibrada de la probeta. Este fenómeno es encontrado en la mayoría de los plásticos amorfos vidriosos, a menudo pueden llevar error con el crecimiento de una red interna de pequeñas grietas. 


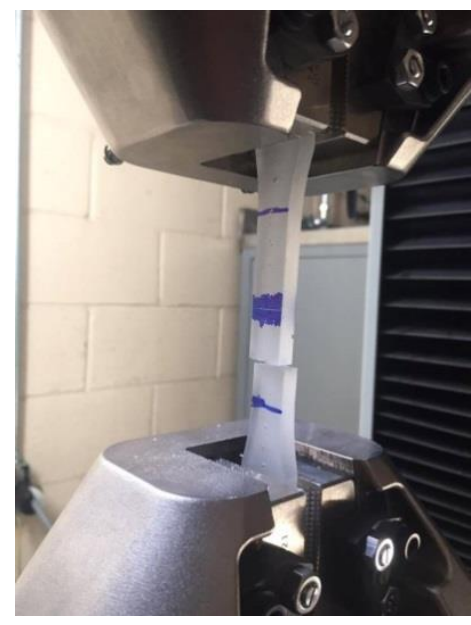

Figura 8: Probeta Tipo I sometida a prueba de tensión.

Los especímenes de material compuesto al ser sometidos a pruebas de tensión presentaron fallas laterales a lo ancho de las probetas, delaminaciones y fallas a $45^{\circ}$, todas dentro del área calibrada. Un ejemplo de ello fue la probeta 2 , así como se muestra en la figura 9.

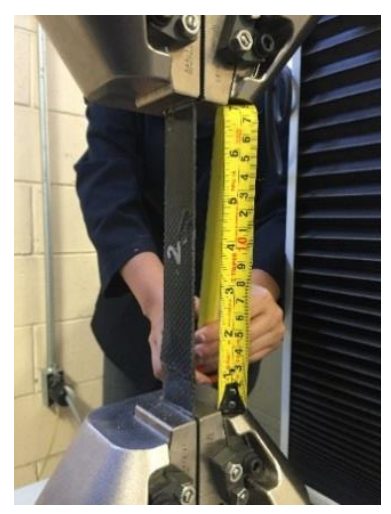

Figura 9: Probeta de material compuesto, sometida a prueba de tensión.

\subsubsection{Microscopia en matriz polimérica}

Una vez realizadas las pruebas de tensión en los especímenes de matriz polimérica tipo I, estos fueron sometidos a observación en el punto de ruptura en un Microscopio Mitutoyo MSM-414L con magnificación de 40X [10]. Los daños causados por las pruebas de tensión se exhiben en la zona calibrada de los especímenes, en donde se presentan burbujas en el interior del material.

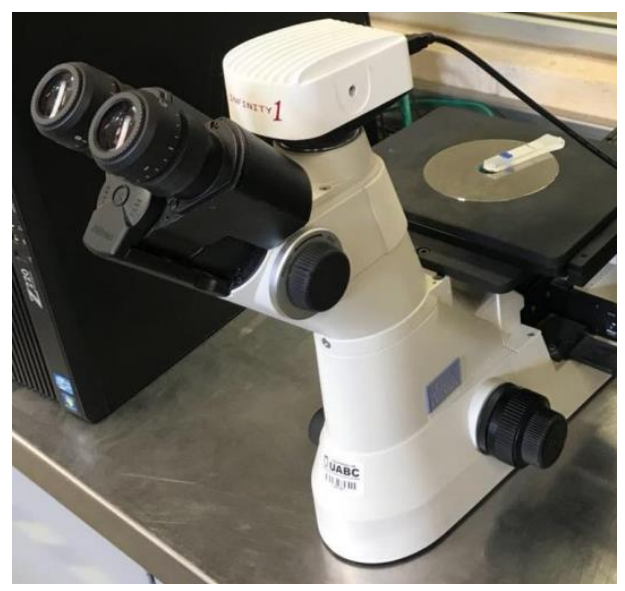

Figura 10: Muestra de probeta de resina epóxica, examinada en microscopio 


\section{RESULTADOS Y DISCUSIÓN}

\subsection{Matriz polimérica}

Los primeros resultados se enfocan en la obtención de valores máximos de esfuerzos en las probetas tipo I de resina epóxica (sin refuerzo de fibra de carbono), los cuales se presentan en la tabla 2.

Tabla 2: Resultados máximos de esfuerzo-deformación para resina epóxica

\begin{tabular}{c|c|c}
\hline $\begin{array}{c}\text { RESINA EPÓ- } \\
\text { XICA }\end{array}$ & ESFUERZO (MPA) & DEFORMACIÓN (\%) \\
\hline Probeta 1 & 182,2 & $5,42 \%$ \\
\hline Probeta 2 & 138,7 & $5,03 \%$ \\
\hline Probeta 3 & 160,4 & $5,49 \%$ \\
\hline Probeta 4 & 140,9 & $4,34 \%$ \\
\hline Probeta 5 & 160,42 & $5,49 \%$ \\
\hline
\end{tabular}

Comparando los resultados de la tabla 2 la probeta 1 muestra una superioridad mecánica más elevada, a pesar de que exhibe un porcentaje de deformación elevado. Cabe mencionar que durante las pruebas de tracción existió un comportamiento normal de alineación y de carga en los especímenes. Los resultados máximos de esfuerzo y deformación que se presentan en la tabla, se muestran en la gráfica 1: curva esfuerzo deformación.

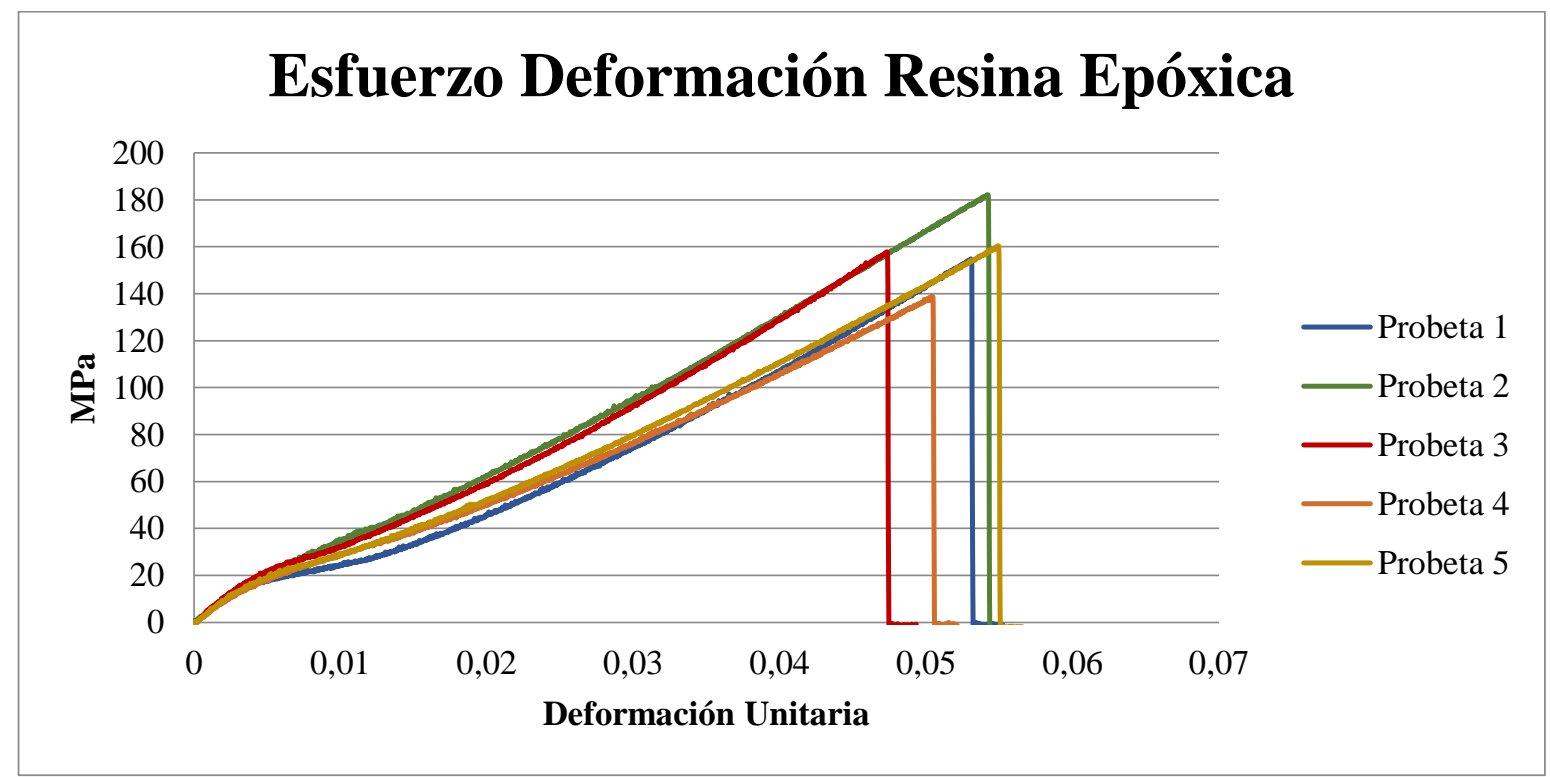

Gráfica 1: Curvas Esfuerzo-Deformación de matriz polimérica (Resina Epóxica)

Como se muestra en la gráfica 1 de Esfuerzo-Deformación de la matriz termoestable, indica que la resina epóxica se comporta como un polímero duro y tenaz, ya que experimenta un alargamiento moderado antes del punto de fluencia, seguido de la deformación irreversible. En la zona elástica el material se comporta como un vidrio, en donde la deformación es permanente.

Posterior a los ensayos de tensión aplicados en los especímenes de resina epóxica se prosiguió a observar las fallas de un punto de resquebrajamiento. La zona afectada que se muestra en la figura 11 aparece de color blanquecina, la cual es una falla llamada "crazing" que se presenta cuando ocurre una deformación en pruebas de tensión aplicadas a temperatura ambiente. Esta deformación produce cavidades en forma de microhuecos. Así mismo se logra observar a nivel microscópico las grietas que se forman perpendicularmente a la tensión aplicada, estas fallas son llamadas "crazes" [11]. 


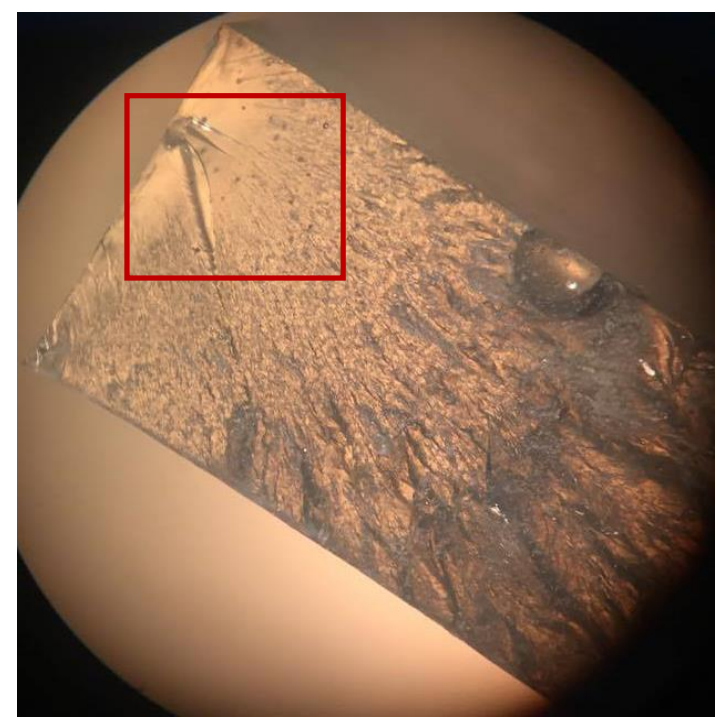

A)

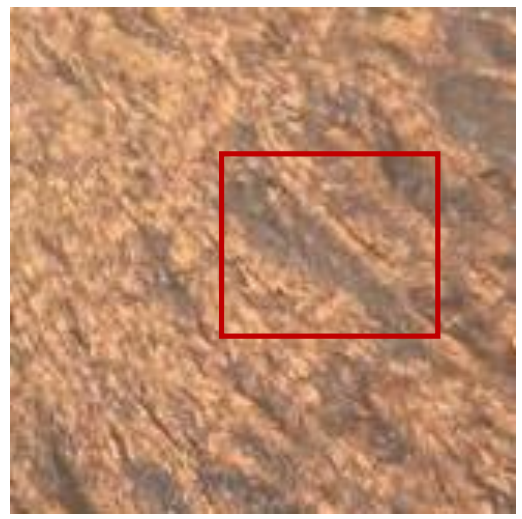

B)

Figura 11: A) Exposición de falla crazing del espécimen 2 a nivel microscópico. B) Grietas crazes perpendiculares a la tensión aplicada.

A pesar que la probeta examinada presentó grietas y microhuecos, se observa de forma clara el concentrador de esfuerzo que llevo al espécimen 2 a su punto de ruptura. La presencia de defectos en forma discontinua dentro de la matriz polimérica provoca un incremento de las tenciones, a los cuales se les llama "concentradores de esfuerzos", mismo que ocasionan que los especímenes lleguen a su punto de ruptura antes de sobrepasar a su límite elástico.

\subsection{Material Compuesto}

Los esfuerzos máximos soportados en pruebas de tracción de los especímenes de material compuesto se presentan en la tabla 3. Donde se muestra una superioridad de esfuerzo en la probeta 4, a pesar que en las 5 muestras se observa una correlación tanto en el esfuerzo y la deformación.

Tabla 3: Valores máximos de esfuerzos sometidos y porcentaje de deformación en especímenes material compuesto.

\begin{tabular}{c|c|c}
\hline $\begin{array}{c}\text { MATERIAL COMPUES- } \\
\text { TO }\end{array}$ & ESFUERZO (MPA) & DEFORMACIÓN (\%) \\
\hline Probeta 1 & 237,6 & $3,38 \%$ \\
\hline Probeta 2 & 225,1 & $3,62 \%$ \\
\hline Probeta 3 & 243,3 & $3,49 \%$ \\
\hline Probeta 4 & 249,3 & $3,22 \%$ \\
\hline Probeta 5 & 233,0 & $3,17 \%$ \\
\hline
\end{tabular}

En la gráfica 2 se observa el efecto de la curva esfuerzo-deformación sobre la resistencia a la tensión en las muestras de material compuesto. La superioridad de los esfuerzos máximos soportados se debe a la orientación de la fibra de carbono [0/ \pm 45$] \mathrm{s}$, en el material compuesto, la cual actúa efectivamente como el material de refuerzo de la resina epóxica. 


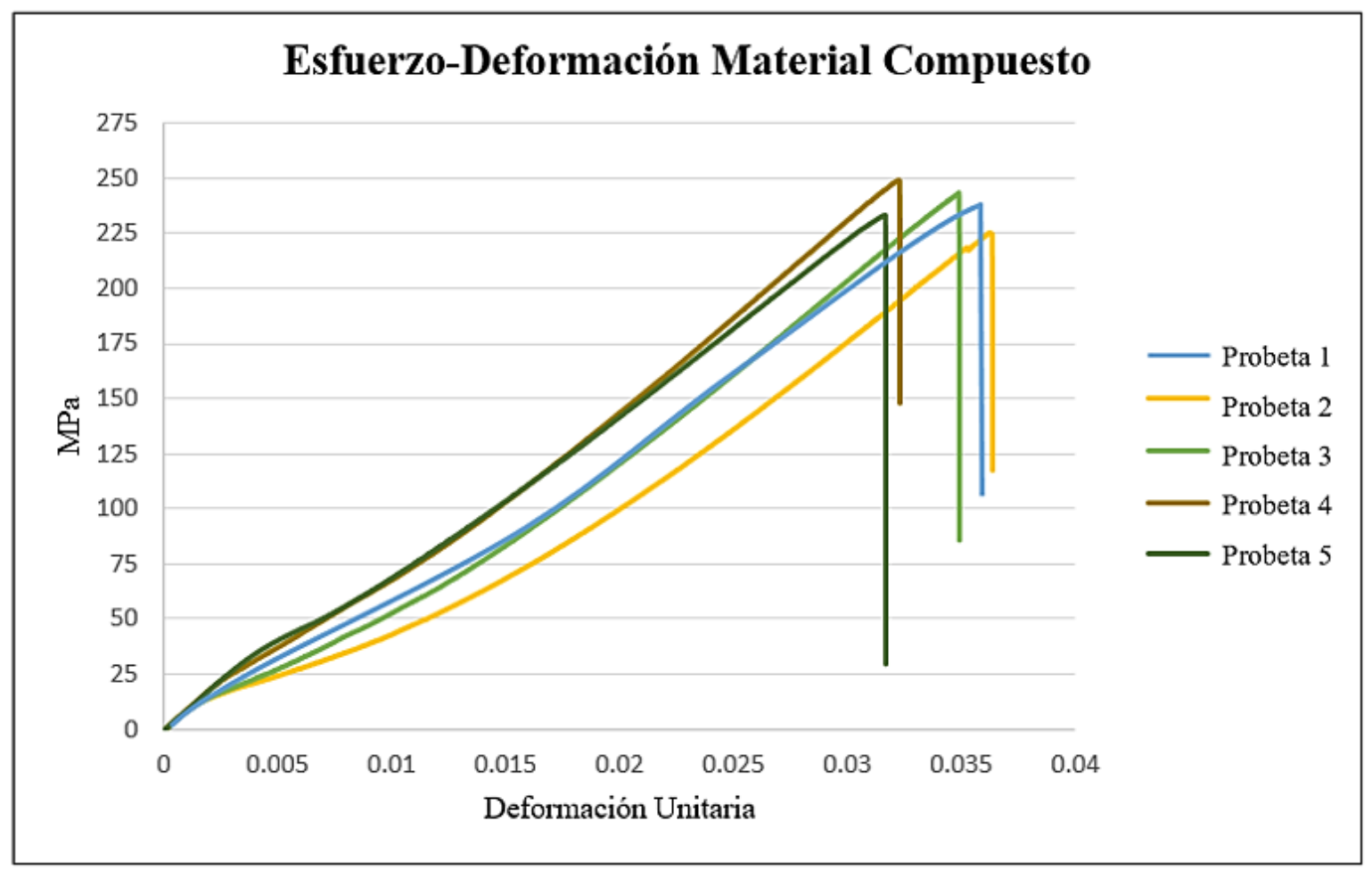

Gráfica 2: Esfuerzo-Deformación de material compuesto

En el material compuesto, durante los ensayos de tensión, los esfuerzos aplicados son más elevados, ya que, al contener fibra de carbono, esta trabaja en paralelo a las cargas aplicadas, lo cual permite distribuirlas uniformemente a través de los filamentos de fibra de carbono, así como lo menciona ROCHA-RANGEL et al. [12]. Las fracturas ocasionadas en los especímenes se dieron en puntos similares dentro de la zona calibrada y a un ángulo $45^{\circ}$, tal como se observa en la figura 12; donde se exhibe el esfuerzo máximo soportando en los especímenes, el cual no da lugar a una fractura abrupta y a una separación completa de las muestras.

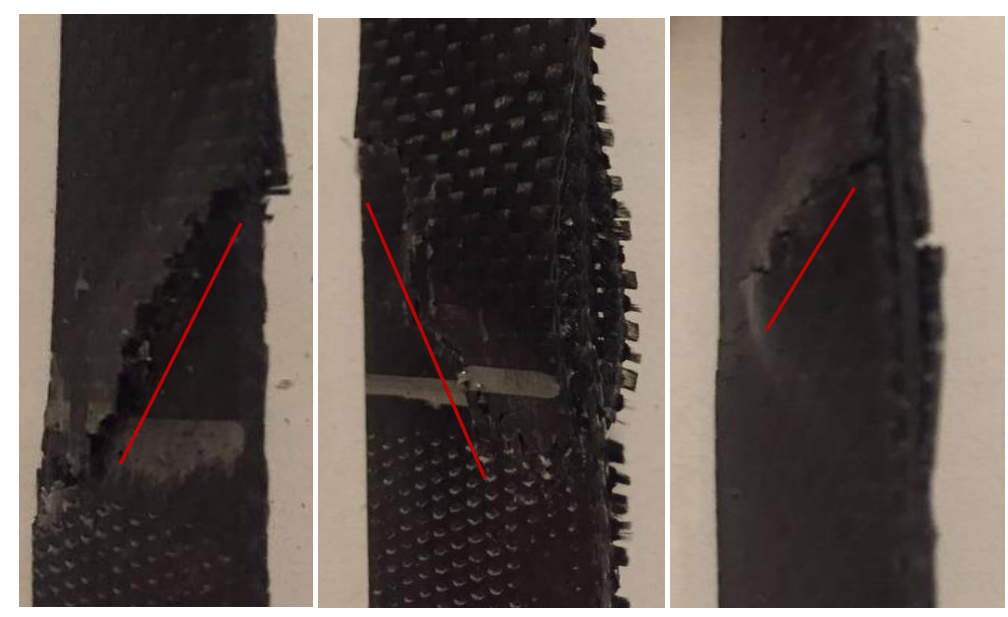

Figura 12: Fractura de 3 probetas ensayadas por tención.

\subsection{Comparación de los materiales}

El comportamiento de los especímenes de resina epóxica sometidos a pruebas de tensión es de clase hookeano [11] antes del punto de fluencia. Lo cual afirma que el esfuerzo aplicado es proporcional a la deformación resultante. Los resultados promedios se presentan en la tabla 4, donde se exhibe el módulo de Young $(E)$, el esfuerzo máximo $(\sigma)$ y el porcentaje de elongación $(\varepsilon)$. 
Tabla 4: Valores promedio de los resultados mecánicos de pruebas de tensión.

\begin{tabular}{c|c|c|c}
\hline PROPIEDADES MECÁNICAS & RESINA EPÓXICA & MATERIAL COMPUESTO & PORCENTAJE DE MEJORA \\
\hline E & $499 \mathrm{MPa}$ & $863 \mathrm{MPa}$ & $72 \%$ \\
\hline$\sigma$ máx. & $156,22 \mathrm{MPa}$ & $238 \mathrm{MPa}$ & $52,35 \%$ \\
\hline$\varepsilon$ máx. & $5,15 \%$ & $3,38 \%$ & $-34,36 \%$ \\
\hline
\end{tabular}

Tal como se presentan los resultados en la tabla 4, el módulo de elasticidad y los esfuerzos máximos del material compuesto aumenta significativamente en comparación con la resina epóxica, esto se debe a que las mejoras de las propiedades mecánicas del material residen en la fibra de carbono. El aporte de la fibra de carbono en la matriz polimérica ayuda a disminuir el porcentaje de deformación, disminuyendo un 34,26 \%, evitando que el material compuesto llegue a una ruptura completa, provocando la separación de los especímenes.

\subsection{Análisis Estadístico}

El análisis estadístico es basado en una prueba de Hipótesis, donde se propone un $95 \%$ de confiabilidad y un error del 5\%. Proponiendo así los puntos siguientes:

H0: Hipótesis Nula proponen que la media del material compuesto es igual a la media de la matriz polimérica. ( $\bar{X}$ Material Compuesto $=\bar{X}$ Resina Epóxica).

H1: Hipótesis alternativa propone que la media del material compuesto será mayor que la media de la matriz polimérica $(\bar{X}$ Material Compuesto $>\bar{X}$ Resina Epóxica).

Tabla 5: Datos del análisis estadístico.

\begin{tabular}{c|c|c|c|c|c}
\hline MATERIAL & $\begin{array}{c}\text { NÚMERO DE } \\
\text { ESPECÍMENES }\end{array}$ & MEDIA $[\overline{\boldsymbol{X}}]$ & $\begin{array}{c}\text { DESVIACIÓN ESTÁN- } \\
\text { DAR }[\mathrm{S}]\end{array}$ & $\begin{array}{c}\text { ERROR ESTÁNDAR DE } \\
\text { LA MEDIA }\end{array}$ & \multirow{2}{*}{ VALO P [P] } \\
\hline $\begin{array}{c}\text { Material } \\
\begin{array}{c}\text { Compues- } \\
\text { to }\end{array}\end{array}$ & 5 & 862,61 & 350,7 & 156,8 & \multirow{2}{*}{0,000} \\
\hline $\begin{array}{c}\text { Resina } \\
\text { Epóxica }\end{array}$ & 5 & 499,01 & 238,5 & 106,6 & \\
\hline
\end{tabular}

El valor utilizado para determinar si los resultados estadísticos son significativos de acuerdo a la prueba de hipótesis, es el valor P que se muestra en la tabla 5, el cual al ser menor que 0.05, por el 5\% de error, establece el rechazo evidencial de la hipótesis nula. Por lo tanto, se puede decir que estadísticamente el material compuesto es más resistente y contiene un módulo de elasticidad más elevado en comparación con la resina epóxica; aceptando el planteamiento de la hipótesis alternativa. 


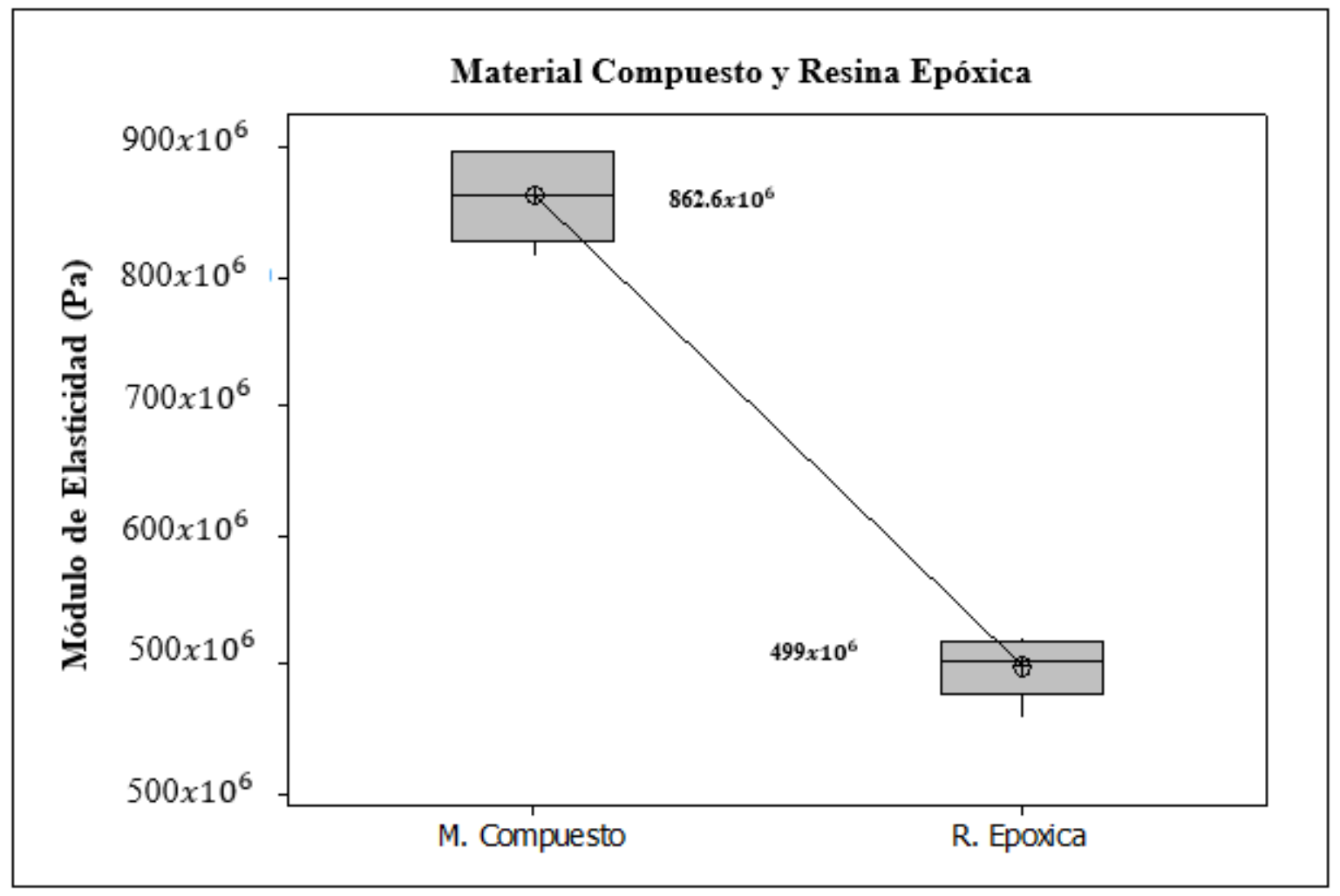

Gráfica 3: Gráfica comparativa del módulo de elasticidad promedio del material compuesto y la de su matriz polimérica.

El análisis muestra características superiores representativas para el material compuesto cuasi isotrópico, en comparación al de su matriz polimérica, ya que presenta una mejora del $72 \%$ en el módulo de elasticidad tal como se muestra en la tabla 4.

En general los resultados obtenidos son aceptables, ya que se comprueba que las propiedades mecánicas del material compuesto aumentan significativamente respecto a la matriz polimérica; esto se debe a la homogeneidad de los valores obtenidos en las pruebas de tracción y en los análisis estadísticos. El nivel de esfuerzos se ve mejorado por la fibra de carbono con un 52\%, aumentando la resistencia del material compuesto.

\section{CONCLUSIONES}

La comparación de las propiedades mecánicas del estudio presentado, muestra que primeramente en las pruebas realizadas en la matriz polimérica de resina epóxica el módulo de elasticidad es una constante que se presenta en el material, pero debido al curado del material, este se comporta como un polímero frágil con características de un vidrio. Es posible que en la matriz se generen microhuecos que funcionen como concentradores de esfuerzos, los cuales provocan que el material falle antes de llegar a su punto de ruptura dentro de su zona elástica, esto se debe a la dificultad de controlar en el proceso de manufactura el curado de la resina epóxica.

En cambio, el comportamiento del material compuesto en comparación al de su matriz polimérica, se muestra beneficiado por la implementación de fibra de carbono, la cual funciona como el refuerzo aceptando parte de las cargas aplicadas a la matriz polimérica; así mismo la geometría del laminado ayuda a distribuir los esfuerzos a $45^{\circ}$, evitando el desprendimiento del material. Otro factor contribuyente a las mejoras de las propiedades mecánicas del material compuesto, fue el método de manufactura empleado, el cual, al aplicar el vacío, este disminuye la formación de concentradores de esfuerzos en la matriz polimérica; se comprueba que el refuerzo del material compuesto reside en las fibras de carbono, ya que la influencia de implementación de capas de fibra de carbono aumenta el módulo de elasticidad y reduce el porcentaje de deformación del material compuesto. 


\section{AGRADECIMENTOS}

A la Escuela de Ciencias de la Ingeniería y Tecnología de la Universidad Autónoma de Baja California, por permitirnos utilizar sus instalaciones y equipo que fue empleado para la realización de este estudio.

\section{BIBLIOGRAFÍA}

[1] GUERRERO, V.H.. "Materiales Compuestos", In: Nuevos Materiales: Aplicaciones Estructurales e Industriales, 1 ed., chapter 2, Quito - Ecuador, imprefepp, Dic. 2011.

[2] GARCÍA CASTILLO, S.K., Análisis de laminados de materiales compuestos con precarga en su plano y sometidos a impacto, Tesis de D.Sc., Universidad Carlos III de Madrid, Leganés, España, 2007.

[3] MENDOZA-NAVA, H. "Caracterización de materiales compuestos reforzados con fibra de carbono fabricados por el método de infusión para aplicaciones aeronáuticas", In: Memorias del XX Congreso Internacional Anual de la SOMIM, pp.838-845, Querétaro, México, Septiembre 2014.

[4] RAMOS J., "Comportamiento mecánico de compuestos en matriz con resina epóxica y resina poliéster reforzados con fibra de carbono unidireccional", In: Congreso Nacional de Metalurgia y Materiales/SAM, Santiago Chile, Nov 2008.

[5] FIBRE GLAST, http://www.fibreglast.com/product/System-1000-Epoxy. Accedido en Octubre de 2017.

[6] ASTM D638-03, "Standard Test Method for Tensile Properties of Plastics", In: ASTM INTERNATIONAL, pp.1-15, 2003.

[7] ASTM D3039/D 3039 M-00, "Standard Test Method for Tensile Propieties of Polymer Matrix Composite", In: ASTM INTERNATIONAL, v.08.01, ASMT Institute for Standard Resarch United States, pp. 105116, 1997

[8] SIQUEIROS-HERNANDEZ, M. "Comparative Analysis in a straight section of an orthogonal symetric balanced composite with aluminum 6061 T6", Journal of Applied Sciences, v. 15, pp. 1223-1230, 2015.

[9] RODRIGUES, J. "Polimeric composites reinforced with natural fibers from Amazon manufactured by infusion", revista Matéria, v.20, n.4, pp. 946-960, 2015.

[10] MITUTOYO USA, http://ecatalog.mitutoyo.com/. Accedido en Octubre de 2017.

[11] ÁLVAREZ, F.B., Resistencia de Polímeros, In:Curso Materiales No Metalicos, Universidad de Oviedo, Escuela de Minas, España, 2014.

[12] ROCHA-RANGEL, E. "Estudio de la Resistencia Mecánica de Materiales Compuestos Poliméricos Reforzados con Fibra de Carbono.", In: Avances en Ciencias e Ingeniería (Executive Business School Chile ,v. 2, n.4, pp.81-88, 2011. 